\title{
Maximum Wind Power Tracking of Doubly Fed Wind Turbine System Based on Adaptive Gain Second-Order Sliding Mode
}

\author{
Hongchang Sun $\mathbb{D}^{1},{ }^{1}$ Yaozhen Han ${ }^{D},{ }^{2}$ and Lvyuan Zhang $^{3}$ \\ ${ }^{1}$ Shandong Dawei International Architectural Design Co., Ltd., Jinan 250061, China \\ ${ }^{2}$ School of Information Science and Electrical Engineering, Shandong Jiaotong University, Jinan, Shandong 250357, China \\ ${ }^{3}$ Shandong Jinan Langchao Electronic Information Industry Group Corp. Co., Ltd., Jinan 250000, China \\ Correspondence should be addressed to Yaozhen Han; yzhan_sdjtu@163.com
}

Received 11 August 2017; Revised 20 November 2017; Accepted 5 December 2017; Published 29 January 2018

Academic Editor: Carlos-Andrés García

Copyright (C) 2018 Hongchang Sun et al. This is an open access article distributed under the Creative Commons Attribution License, which permits unrestricted use, distribution, and reproduction in any medium, provided the original work is properly cited.

\begin{abstract}
This paper proposes an adaptive gain second-order sliding mode control strategy to track optimal electromagnetic torque and regulate reactive power of doubly fed wind turbine system. Firstly, wind turbine aerodynamic characteristics and doubly fed induction generator (DFIG) modeling are presented. Then, electromagnetic torque error and reactive power error are chosen as sliding variables, and fixed gain super-twisting sliding mode control scheme is designed. Considering that uncertainty upper bound is unknown and is hard to be estimated in actual doubly fed wind turbine system, a gain scheduled law is proposed to compel control parameters variation according to uncertainty upper bound real-time. Adaptive gain second-order sliding mode rotor voltage control method is constructed in detail and finite time stability of doubly fed wind turbine control system is strictly proved. The superiority and robustness of the proposed control scheme are finally evaluated on a $1.5 \mathrm{MW}$ DFIG wind turbine system.
\end{abstract}

\section{Introduction}

In the 21st century, as human population grows and nonregeneration energy is rapidly consumed, climate warming and environmental degradation problems become increasingly serious. Renewable energy such as wind energy, hydroenergy, and nuclear energy is highly favored. Particularly, wind energy, as one of the clean renewable energy sources, is attracting more and more attention [1]. The newly installed capacity of wind power reaches $54.6 \mathrm{GW}$ in 2016 [2].

Among the many adopted wind power technologies, doubly fed induction generator (DFIG) has been widely used in the wind power industry and dominated the market due to its excellent operating performances such as light weight, small size, highly cost effective, and small capacity of converter. In order to enhance generating efficiency, wind energy conversion optimization is one of the key issues in the DFIG wind power generation control system $[3,4]$.

Many scholars study various control methods for optimizing wind energy conversion [5]. However, due to the randomness of wind, nonlinearity and uncertainty of generator set, it is hard to achieve wind energy capture rapidly and accurately via linear control theory. Nonlinear control techniques such as fuzzy logic [6], neural networks [7], feedback linearization [8], and backstepping control [9] are widely employed to achieve optimal conversion of wind power system. Despite the recognized advantage of using nonlinear controllers to cope with nonlinear systems, many of these techniques produce control laws with a rather high computational burden. These calculi commonly depend on the system states and also on several model parameters having the secondary effect of reducing control robustness. Other controllers, like fuzzy and neural networks methods, present the drawback of neither being systematic in the design stage nor providing a rigorous stability proof.

Sliding mode control is a nonlinear robust control method and has already successfully applied to doubly fed wind turbine system [10-12]. However, as an on-off control, control effect of conventional first-order sliding mode is discontinuous and chattering phenomenon is serious. These drawbacks inevitably increase mechanical and electrical wear and shorten service life. 
Higher-order sliding mode can cancel the requirement of compelling relative degree to be one, enhance sliding accuracy, attenuate chattering phenomenon, and make control effective continuous [13]. Super-twisting algorithm, called higher-order sliding mode of the second order, has been adopted to achieve maximum wind power tracking and reactive power regulation [14-19]. However, control gains for these schemes cannot be real-time adjusted along with variation of system uncertainty. The rotor current or rotor voltage is conservatively chosen, and this will aggravate control chattering. Paper [20] proposes an adaptive secondorder sliding mode control strategy to maximize the energy production simultaneously reducing the mechanical stress on the shaft. Yet, preliminary results of this proposal are applied to a simple single-input single-output topology and based on a unidirectional DFIG. Paper [21] presents a novel adaptive higher-order sliding mode control strategy for DFIG energy conversion system. Adaptive natures of the gains formulate a zero steady state transient performance of the system gifted by a reduced settling time. However, the system cannot converge in finite time. Evangelista et al. [22] achieve adaptive secondorder sliding mode control for doubly fed wind turbine in finite time based on receding horizon adaptation time windows algorithm. Yet, the selection of adaptation period parameter should be kept in mind while tuning the whole control system.

In this context, to face the stringent specifications of maximum wind power tracking and reactive power control problems of doubly fed wind turbine system where the uncertainties are subjected to fast variations, this paper proposes a new adaptive control gain second-order sliding mode scheme. It is not necessary to know the upper bound of uncertainty in advance by choosing suitable sliding function and designing adaptive gain second-order sliding mode controller. Control parameters can be adjusted according to variation of uncertainty upper bound. The control objectives of maximum wind power tracking and reactive power regulation are achieved by controlling rotor voltage, and the output chattering is greatly restrained.

This paper is organized as follows. Section 2 presents system modeling of doubly fed wind turbine. The detailed fixed gain and adaptive gain control strategies are explained in Section 3. The proposed control strategy is validated in MATLAB environment which is illustrated in Section 4.

\section{Doubly Fed Wind Turbine System Modeling}

Structure diagram of doubly fed wind turbine system is shown in Figure 1, which mainly includes wind turbine, gearbox, DFIG, and converter. Firstly, wind energy is converted to mechanical energy via wind turbine, and then mechanical energy is converted to electric energy by means of DFIG. DFIG stator is directly connected to grid, and rotor is by way of converter. DFIG can feed into the grid via stator and rotor under hypersynchronous mode.

According to Betz' Law, the captured mechanical power of wind turbine is

$$
P_{a}=\frac{1}{2} \pi \rho R^{2} C_{p}(\lambda, \beta) v^{3},
$$

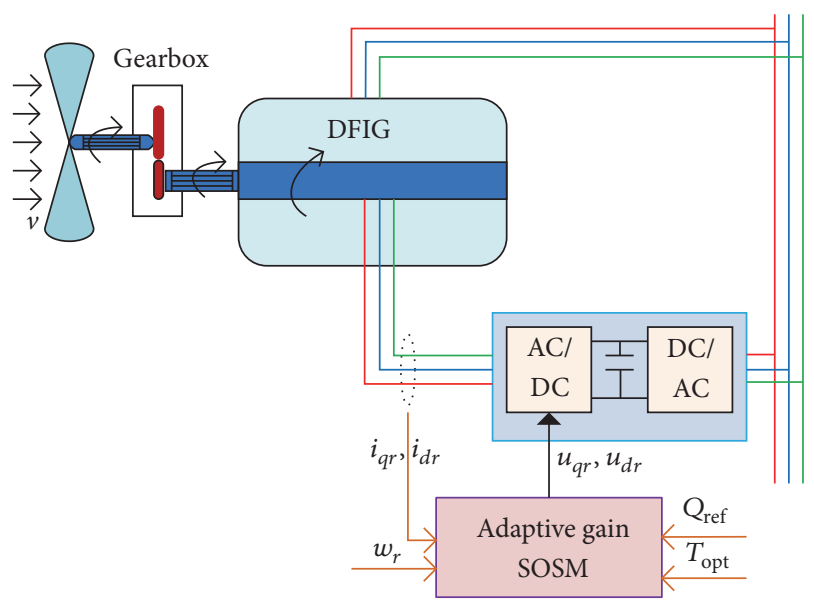

FiguRE 1: Schematic diagram of doubly feed wind turbine system.

where $\rho$ is air density $\left(\mathrm{kg} / \mathrm{m}^{3}\right), R$ represents blade radius $(\mathrm{m})$, $v$ is wind speed $(\mathrm{m} / \mathrm{s})$, and $C_{p}$ is power conversion efficiency which is nonlinear function of tip speed ratio $\lambda$ and pitch angle $\beta$. Tip speed ratio $\lambda$ is defined as

$$
\lambda=\frac{\omega_{m r} R}{v}=\frac{\omega_{r} R}{k_{g b} v}
$$

where $\omega_{m r}$ is rotating speed $(\mathrm{rad} / \mathrm{s}), \omega_{r}$ is DFIG rotor speed, and $k_{g b}$ is gear ratio.

Power conversion efficiency $C_{p}$ can be expressed as [23]

$$
C_{p}(\lambda, \beta)=c_{1}\left(c_{2} \frac{1}{\lambda_{s}}-c_{3} \beta-c_{4}\right) e^{-c_{5} / \lambda_{s}}+c_{6} \lambda,
$$

where $1 / \lambda_{s}=1 /(\lambda+0.08 \beta)-0.035 /\left(1+\beta^{3}\right), c_{1}=0.5176$, $c_{2}=116, c_{3}=0.4, c_{4}=5, c_{5}=21$.

Wind turbine mechanical power can also be defined as

$$
P_{a}=T_{a} \omega_{m r}
$$

Then, wind turbine mechanical torque is

$$
T_{a}=\frac{P_{a}}{\omega_{m r}}=\frac{1}{2 k_{g b}} \pi \rho R^{3} C_{t}(\lambda, \beta) v^{2}
$$

where $C_{t}(\lambda, \beta)=C_{p}(\lambda, \beta) / \lambda$ is wind turbine torque coefficient.

Dynamic characteristics of doubly fed wind turbine system can be accurately described via fifth-order differential equations [24], four of which are expressed by flux linkage under $d-q$ synchronous rotating coordinate axis:

$$
\begin{aligned}
& \dot{\psi}_{d s}=u_{d s}-R_{s} i_{d s}+\omega_{s} \psi_{q s}, \\
& \dot{\psi}_{q s}=u_{q s}-R_{s} i_{q s}-\omega_{s} \psi_{d s}, \\
& \dot{\psi}_{d r}=u_{d r}-R_{r} i_{d r}+\left(\omega_{s}-p \omega_{r}\right) \psi_{q r}, \\
& \dot{\psi}_{q r}=u_{q r}-R_{r} i_{q r}-\left(\omega_{s}-p \omega_{r}\right) \psi_{d r},
\end{aligned}
$$


where

$$
\begin{aligned}
& \psi_{d s}=L_{s} i_{d s}+L_{m} i_{d r}, \\
& \psi_{q s}=L_{s} i_{q s}+L_{m} i_{q r}, \\
& \psi_{d r}=L_{r} i_{d r}+L_{m} i_{d s}, \\
& \psi_{q r}=L_{r} i_{q r}+L_{m} i_{q s} .
\end{aligned}
$$

In formulas (6) and (7), subscripts $s$ and $r$ denote stator and rotor physical quantities. $d$ and $q$ are physical quantities of $d$-axis and $q$-axis. $u, i, r, \psi$ denote voltage, current, resistance, and flux linkage, respectively, $\omega_{s}$ is power grid angular frequency, $L_{s}$ and $L_{r}$ are self-inductance of stator winding and rotor winding, and $L_{m}$ is mutual inductance of stator and rotor.

The fifth differential equation is kinematic equation:

$$
\dot{\omega}_{r}=\frac{1}{J}\left(T_{a}-T_{e}\right)
$$

where $J$ represents inertia of the whole rotating part and $T_{e}$ is DFIG electromagnetic torque:

$$
T_{e}=\frac{3}{2} p L_{m}\left(i_{q s} i_{d r}-i_{d s} i_{q r}\right)
$$

where $p$ is number of pole pairs.

For achieving decoupling control, $d$-axis under synchronous rotating coordinate system is oriented to stator flux linkage vector and stator resistance is neglected. Reducedorder model of doubly fed wind turbine system can be deduced from formula (5) to formula (9):

$$
\begin{aligned}
\dot{i}_{q r}= & -\left(\frac{L_{m} V_{s}}{L_{e q}}+\omega_{s} i_{d r}\right)\left(1-\frac{p}{\omega_{s}} \omega_{r}\right)-\frac{R_{r} L_{s}}{L_{e q}} i_{q r} \\
& +\frac{L_{s}}{L_{e q}} u_{q r}, \\
\dot{i}_{d r}= & \omega_{s} i_{q r}\left(1-\frac{p}{\omega_{s}} \omega_{r}\right)-\frac{R_{r} L_{s}}{L_{e q}} i_{d r}+\frac{L_{s}}{L_{e q}} u_{d r}, \\
\dot{\omega}_{r}= & \frac{1}{J}\left(T_{a}-\frac{3 p L_{m} V_{s}}{2 \omega_{s} L_{s}} i_{q r}\right),
\end{aligned}
$$

where $V_{s}$ is network line voltage, $L_{e q}=L_{s} L_{r}-L_{m}^{2}$. Then stator current is calculated as

$$
\begin{aligned}
& i_{q s}=-\frac{L_{m}}{L_{s}} i_{q r}, \\
& i_{d s}=\frac{V_{s}}{\omega_{s} L_{s}}-\frac{L_{m}}{L_{s}} i_{d r} .
\end{aligned}
$$

Reactive power is expressed as

$$
Q_{s}=\frac{3 V_{s}^{2}}{2 \omega_{s} L_{s}}-\frac{3 L_{m} V_{s}}{2 L_{s}} i_{d r}
$$

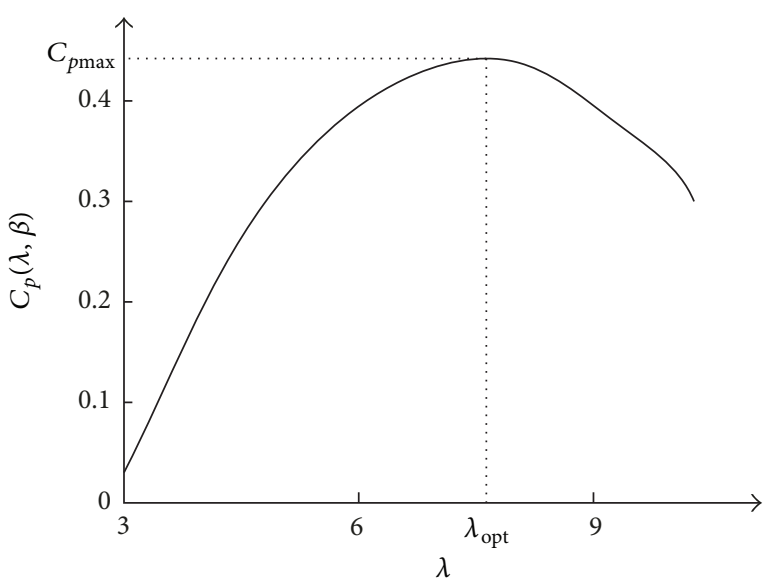

FIGURE 2: The relation between power coefficient and tip speed ratio.

\section{Adaptive Gain Higher-Order Sliding Mode Control Strategy}

3.1. Control Objective Description. In the constant power zone, the objective is to adjust pitch angle to maintain wind turbine system operating at rated power, while, in the partial load zone, the objective is to track maximum wind energy. Control objectives of this paper are to achieve maximum wind power tracking and reactive power regulation according to grid demand and tolerance range of wind power system.

The pitch angle $\beta$ is set as zero when wind turbine system operates at the partial load zone. Then wind turbine power coefficient $C_{p}$ is only related to tip speed ratio $\lambda$. As is shown in Figure 2, there is always $C_{p \max }$ related to optimal tip speed ratio $\lambda_{\text {opt }}$ for certain wind turbine. In other words, wind turbine can only operate under specific revolving speed to achieve maximum wind energy conversion efficiency for specific wind speed.

When wind turbine operates at maximum power point $\left(C_{p}=C_{p \max }, \lambda=\lambda_{\text {opt }}\right)$, the corresponding torque is expressed as

$$
T_{\mathrm{opt}}=\frac{\pi \rho R^{5} C_{p \max }}{2 k_{g b}^{3} \lambda_{\mathrm{opt}}^{3}} \omega_{r}^{2}=k_{0} \omega_{r}^{2},
$$

where $k_{0}=\pi \rho R^{5} C_{p \max } / 2 k_{g b}^{3} \lambda_{\text {opt }}^{3}$.

The objective of maximum wind power tracking control is further presented as achieving $T_{e}=T_{\text {opt }}$ for any wind speed at partial load zone. Considering power regulation ability enhancement and power system stability improvement, the other control objective is reactive power regulation. The choice of reactive power reference value mainly relies on certain evaluation function to attain the optimal one.

3.2. Controller Design. For doubly fed wind turbine system (10), this section firstly presents standard super-twisting second-order sliding mode design method. Then considering unknown uncertainty upper bound and system wear reduction, adaptive gain second-order sliding mode control scheme is detaily designed to achieve maximum wind power 
tracking and reactive power regulation. Finite time stability of the closed-loop system is also strictly analyzed and proved.

3.2.1. Design Procedure under Fixed Control Gain. For tracking maximum wind power and regulating reactive power, the sliding variables are chosen as

$$
\begin{aligned}
& s_{1}=T_{\mathrm{opt}}-T_{e}=k_{0} \omega_{r}^{2}-\frac{3 p L_{m} V_{s}}{2 \omega_{s} L_{s}} i_{q r}, \\
& s_{2}=Q_{\mathrm{ref}}-Q=Q_{\mathrm{ref}}-\frac{3 p V_{s}}{2 L_{s}}\left(\frac{V_{s}}{\omega_{s}}-L_{m} i_{d r}\right) .
\end{aligned}
$$

To calculate first-order time derivative of $s_{1}$ and $s_{2}$

$$
\begin{aligned}
\dot{s}_{1} & =2 k_{0} \omega_{r} \dot{\omega}_{r}-\frac{3 p L_{m} V_{s}}{2 \omega_{s} L_{s}} i_{q r} \\
& =f_{1}\left(t, i_{q r}, i_{d r}, \omega_{r}\right)+g_{1}\left(t, i_{q r}, i_{d r}, \omega_{r}\right) u_{q r}, \\
\dot{s}_{2} & =\dot{Q}_{\mathrm{ref}}+\frac{3 p L_{m} V_{s}}{2 \omega_{s} L_{s}} i_{d r} \\
& =f_{2}\left(t, i_{q r}, i_{d r}, \omega_{r}\right)+g_{2}\left(t, i_{q r}, i_{d r}, \omega_{r}\right) u_{d r},
\end{aligned}
$$

where

$$
\begin{aligned}
f_{1}\left(t, i_{q r}, i_{d r}, \omega_{r}\right) & =\frac{3 p L_{m} V_{s}}{2 \omega_{s} L_{s}}\left(\frac{L_{m} V_{s}}{L_{e q}}+\frac{R_{r} L_{s}}{L_{e q}} i_{q r}\right. \\
+\left(\omega_{s}-p \omega_{r}\right) & \left.-\frac{p L_{m} V_{s}}{\omega_{s} L_{e q}} \omega_{r}\right)+\frac{2 k_{0} \omega_{r}}{J}\left(T_{a}-T_{e}\right), \\
g_{1}\left(t, i_{q r}, i_{d r}, \omega_{r}\right) & =-\frac{3 p L_{m} V_{s}}{2 \omega_{s} L_{e q}}, \\
f_{2}\left(t, i_{q r}, i_{d r}, \omega_{r}\right) & =\dot{Q}_{\mathrm{ref}}+\frac{3 p L_{m} V_{s}}{2 L_{s}}\left(\left(\omega_{s}-p \omega_{r}\right)\right. \\
\left.-\frac{R_{r} L_{s}}{L_{e q}} i_{d r}\right) & \\
g_{2}\left(t, i_{q r}, i_{d r}, \omega_{r}\right) & =\frac{3 p L_{m} V_{s}}{2 L_{e q}} .
\end{aligned}
$$

Then there exist positive constants $U_{M i}, C_{i}, \Gamma_{m i}, \Gamma_{M i}, q_{i} \in$ $(0,1)$, satisfying

$$
\begin{aligned}
& \left|\dot{f}_{i}\left(t, i_{q r}, i_{d r}, \omega_{r}\right)\right|+\left|\dot{g}_{i}\left(t, i_{q r}, i_{d r}, \omega_{r}\right)\right| U_{M i} \leq C_{i}, \\
& 0<\Gamma_{m i} \leq\left|g_{i}\left(t, i_{q r}, i_{d r}, \omega_{r}\right)\right| \leq \Gamma_{M i}, \\
& \left|\frac{f_{i}\left(t, i_{q r}, i_{d r}, \omega_{r}\right)}{g_{i}\left(t, i_{q r}, i_{d r}, \omega_{r}\right)}\right|<q_{i} U_{M i},
\end{aligned}
$$

where $i=1,2$. To apply standard super-twisting second-order sliding mode control algorithm

$$
\begin{aligned}
& u_{q r}=-\alpha_{1}\left|s_{1}\right|^{1 / 2} \operatorname{sign}\left(s_{1}\right)+u_{1 q r}, \\
& u_{1 q r}= \begin{cases}-\beta_{1} \operatorname{sign}\left(s_{1}\right) & \left|u_{q r}\right| \leq U_{M 1} \\
-u_{q r} & \left|u_{q r}\right| \leq U_{M 1},\end{cases} \\
& u_{d r}=-\alpha_{2}\left|s_{2}\right|^{1 / 2} \operatorname{sign}\left(s_{2}\right)+u_{1 d r}
\end{aligned}
$$

According to sufficient condition for finite time stability of super-twisting algorithm $[25,26]$, when control parameters satisfy

$$
\begin{aligned}
\Gamma_{m i} \beta_{i} & >C_{i} \\
\alpha_{i} & >\sqrt{\frac{2}{\Gamma_{m i} \beta_{i}-C_{i}}} \frac{\left(\Gamma_{m i} \beta_{i}-C_{i}\right) \Gamma_{M i}\left(1+q_{i}\right)}{\Gamma_{m i}^{2}\left(1-q_{i}\right)}
\end{aligned}
$$

second-order sliding mode with respect to $s_{i}$ can be established in finite time, and optimal torque tracking and reactive power regulation are achieved.

It is observed that uncertainty upper bounds are needed when designing rotor voltage second-order sliding mode controller, yet the upper bounds are usually unknown in practical wind power system and hard to be estimated. Overestimation of these upper bounds may lead to conservative choice of controller parameters which will produce more control effective, aggravate control chattering, and shorten service cycle of wind turbines.

\subsubsection{Adaptive Gain Second-Order Sliding Mode Control} Design. Adaptive control gain can deal with unknown uncertainty upper bound problem and further restrain rotor voltage chattering. Here $u_{q r}$ is redesigned as an example because the system has been decoupled, and design procedure of $u_{d r}$ is similar to $u_{q r}$.

Due to the existence of parameter uncertainty and external disturbance, $f_{1}\left(t, i_{q r}, i_{d r}, \omega_{r}\right)$ and $g_{1}\left(t, i_{q r}, i_{d r}, \omega_{r}\right)$ may be expressed as

$$
\begin{aligned}
& f_{1}\left(t, i_{q r}, i_{d r}, \omega_{r}\right)=\bar{f}_{1}\left(t, i_{q r}, i_{d r}, \omega_{r}\right)+\Delta f_{1}, \\
& g_{1}\left(t, i_{q r}, i_{d r}, \omega_{r}\right)=\bar{g}_{1}\left(t, i_{q r}, i_{d r}, \omega_{r}\right)+\Delta g_{1},
\end{aligned}
$$

where $\Delta f_{1}$ and $\Delta g_{1}$ mainly include fluctuation of $L_{m}, V_{s}, \omega_{s}$, $L_{e q}$ and satisfy

$$
\frac{\left|\Delta g_{1}\right|}{\bar{g}_{1}\left(t, i_{q r}, i_{d r}, \omega_{r}\right)}=\chi_{q}\left(t, i_{q r}, i_{d r}, \omega_{r}\right) \leq \chi_{q 1}<1,
$$

where $\chi_{q 1}$ is unknown upper bound. 
Carrying out feedback control for system (15)

$$
u_{q r}=\bar{g}_{1}^{-1}\left(t, i_{q r}, i_{d r}, \omega_{r}\right)\left(-\bar{f}_{1}\left(t, i_{q r}, i_{d r}, \omega_{r}\right)+v_{q r}\right) ;
$$

then

$$
\begin{aligned}
\dot{\sigma}_{1}= & \Delta f_{1}-\frac{\Delta g_{1}}{\bar{g}_{1}\left(t, i_{q r}, i_{d r}, \omega_{r}\right)} \bar{f}_{1}\left(t, i_{q r}, i_{d r}, \omega_{r}\right) \\
& +\left(1+\frac{\left|\Delta g_{1}\right|}{\bar{g}_{1}\left(t, i_{q r}, i_{d r}, \omega_{r}\right)}\right) v_{q r} \\
= & \Delta f_{1}-\chi_{q}\left(t, i_{q r}, i_{d r}, \omega_{r}\right) \bar{f}_{1}\left(t, i_{q r}, i_{d r}, \omega_{r}\right) \\
& +\left(1+\chi_{q}\left(t, i_{q r}, i_{d r}, \omega_{r}\right)\right) v_{q r}=\tilde{a}_{q}+\tilde{b}_{q} v_{q r},
\end{aligned}
$$

where $\tilde{a}_{q}=\Delta f_{1}-\chi_{q}\left(t, i_{q r}, i_{d r}, \omega_{r}\right) \bar{f}_{1}\left(t, i_{q r}, i_{d r}, \omega_{r}\right)$ and $\tilde{b}_{q}=$ $1+\chi_{q}\left(t, i_{q r}, i_{d r}, \omega_{r}\right)$.

In practice, parameters $L_{m}, V_{s}, \omega_{s}, L_{s}, J$ vary slowly if they are influenced by uncertainties, and $i_{q r}, T_{a}, T_{e}$ are also continuous, such that $\widetilde{a}_{q}$ satisfies

$$
|\dot{\vec{a}}| \leq a_{q 1}
$$

where $a_{q 1}$ exists and is unknown.

Then aiming at formula (26), control law $v_{q r}$ is designed to establish second-order sliding mode with respect to $s_{1}$

$$
\begin{aligned}
v_{q r} & =-\alpha_{q r}|s|^{1 / 2} \operatorname{sign}\left(s_{1}\right)+v_{s m 1}, \\
\dot{v}_{s m 1} & =-\frac{\beta_{q r}}{2} \operatorname{sign}\left(s_{1}\right),
\end{aligned}
$$

where $\alpha_{q r}$ and $\beta_{q r}$ are adaptive control gains.

$\widetilde{b}_{q}$ can be regarded as an uncertain piecewise constant function. Then formulas (26) and (28) may be written as

$$
\begin{aligned}
\dot{s}_{1} & =-\alpha_{q r} \tilde{b}_{q}\left|s_{1}\right|^{1 / 2} \operatorname{sign}\left(s_{1}\right)+\omega_{q *}, \\
\dot{\omega}_{q *} & =-\frac{\beta_{q r} \widetilde{b}_{q}}{2} \operatorname{sign}\left(s_{1}\right)+\dot{\tilde{a}}_{q}+\dot{\widetilde{b}}_{q} v_{s m 1}, \\
\omega_{q *} & =\widetilde{a}_{q}+\widetilde{b}_{q} v_{s m 1}, \\
\omega_{q *}(0) & =0 .
\end{aligned}
$$

$\dot{\widetilde{b}}_{q} v_{s m 1}$ is supposed to have uncertain upper bound $\chi_{q 2}>$ 0 ; meanwhile, control gain $\beta_{q r}$ is bounded, meaning $\left|\beta_{q r}\right| \leq$ $\beta_{q r}^{*}, \beta_{q r}^{*}>0$; then in any finite time interval

$$
\left|\dot{\vec{b}}_{q} \tau_{s m 1}\right| \leq \frac{1}{2}\left|\dot{\vec{b}}_{q}\right| \int_{0}^{t} \beta_{q r} d_{s_{1}} \leq \frac{1}{2}\left|\dot{\vec{b}}_{q}\right| \beta_{q r}^{*} t \leq \chi_{q 2} .
$$

Thus, uncertain function $\dot{\gamma}_{q}=\dot{\tilde{a}}_{q}+\dot{\widetilde{b}}_{q} \tau_{s m 1}$ possesses unknown upper bound; then

$$
\left|\dot{\gamma}_{q}\right| \leq a_{q 1}+\chi_{q 2}=\chi_{q 3} .
$$

Second-order sliding mode control problem of system (26) is converted to design adaptive gain super-twisting algorithm for system (29) to achieve $s_{1} \rightarrow 0, \dot{s}_{1} \rightarrow 0$ in finite time under conditional formulas (24), (27), (30), and (31).

Theorem 1 provides adaptive law of control gain $\alpha_{q r}, \beta_{q r}$, and guarantees establishment of second-order sliding mode with respect to $s_{1}$.

Theorem 1. In order to achieve optimal torque tracking of doubly fed wind power system, q-axis rotor voltage $u_{q r}$ is designed as formula (25), auxiliary control law is formula (28), and adaptive control gain $\alpha_{q r}, \beta_{q r}$ are designed as

$$
\dot{\alpha}_{q r}= \begin{cases}\omega_{q 1} \sqrt{\frac{\delta_{q 1}}{2}} \operatorname{sign}\left(\left|s_{1}\right|-\mu_{q}\right) & \alpha_{q r}>\alpha_{q r m} \\ \eta_{q} & \alpha_{q r} \leq \alpha_{q r m}\end{cases}
$$$$
\beta_{q r}=2 \varepsilon_{q} \alpha_{q r}
$$

then actual second-order sliding mode with respect to $s_{1}$ is established after finite time $t_{f}>0$ and $\alpha_{q r}>\left(2 \chi_{3}+\lambda_{q}+\right.$ $\left.4 \varepsilon_{q}^{2}\right)^{2} / 12 \varepsilon_{q} \lambda_{q}\left(1-\chi_{q 1}\right)-\varepsilon_{q}\left(4 \chi_{3}+1\right) / \lambda_{q}\left(1-\chi_{q 1}\right)$, where $s_{1} \leq \eta_{q 1}$, $\dot{s}_{q 1} \leq \eta_{q 2}, \alpha_{q r m}, \omega_{q 1}, \varepsilon_{q}$ are positive constant.

Proof. To bring in new state vector $\mathbf{z}$

$$
\mathbf{z}=\left[\begin{array}{ll}
z_{1} & z_{2}
\end{array}\right]^{T}=\left[\left|s_{1}\right|^{1 / 2} \operatorname{sign}\left(s_{1}\right) \omega_{q *}\right]^{T} .
$$

Then formula (29) can be expressed as

$$
\begin{aligned}
& \dot{z}_{1}=\frac{1}{2\left|z_{1}\right|}\left(-\alpha_{q r} \tilde{b}_{q} z_{1}+z_{2}\right), \\
& \dot{z}_{2}=-\frac{\beta_{q r} \tilde{b}_{q}}{2\left|z_{1}\right|} z_{1}+\dot{\gamma}_{q} .
\end{aligned}
$$

Formula (34) is rewritten as matrix form

$$
\begin{aligned}
{\left[\begin{array}{c}
\dot{z}_{1} \\
\dot{z}_{2}
\end{array}\right]=} & \frac{1}{2\left|z_{1}\right|}\left[\begin{array}{ccc}
-\alpha_{q r} \tilde{b}_{q} & 1 \\
-\beta_{q r} \tilde{b}_{q} & 0
\end{array}\right]\left[\begin{array}{c}
z_{1} \\
z_{2}
\end{array}\right] \\
& +\frac{1}{2\left|z_{1}\right|}\left[\begin{array}{cc}
1 & 0 \\
0 & 2\left|z_{1}\right|
\end{array}\right]\left[\begin{array}{c}
0 \\
\dot{\gamma}_{q}
\end{array}\right] .
\end{aligned}
$$

Considering $\left|\dot{\tilde{a}}_{q}\right| \leq a_{q 1}$ and $\left|\dot{\gamma}_{q}\right| \leq a_{q 1}+\chi_{q 2}=\chi_{q 3}$, then $\dot{\gamma}_{q}$ is expressed as

$$
\dot{\gamma}_{q}=\frac{\rho_{q 2}}{2} \operatorname{sign}\left(s_{1}\right)=\frac{\rho_{q 2}}{2} \frac{z_{1}}{\left|z_{1}\right|},
$$

where $0<\rho_{q 2} \leq 2 \chi_{q 3}$; then formula (35) is further denoted as

$$
\left[\begin{array}{l}
\dot{z}_{1} \\
\dot{z}_{2}
\end{array}\right]=\overline{\mathbf{A}}\left(z_{1}\right)\left[\begin{array}{l}
z_{1} \\
z_{2}
\end{array}\right],
$$

where $\overline{\mathbf{A}}\left(z_{1}\right)=\frac{1}{2\left|z_{1}\right|}\left[\begin{array}{cc}-\alpha_{q r} \widetilde{b}_{q} & 1 \\ -\beta_{q r} \widetilde{b}_{q}+\rho_{q 2} & 0\end{array}\right]$. 
It is observed that if $z_{1}, z_{2} \rightarrow 0$ in finite time, then $s_{1}, \dot{s}_{1} \rightarrow$ 0 is set up in finite time, and $\left|z_{1}\right|=\left|s_{1}\right|^{1 / 2}, \operatorname{sign}\left(z_{1}\right)=\operatorname{sign}\left(s_{1}\right)$ are both established.

To choose Lyapunov function

$$
\begin{aligned}
V\left(z_{1}, z_{2}, \alpha_{q r}, \beta_{q r}\right)= & V_{0}+\frac{1}{2 \delta_{1}}\left(\alpha_{q r}-\alpha_{q r}^{*}\right)^{2} \\
& +\frac{1}{2 \delta_{2}}\left(\beta_{q r}-\beta_{q r}^{*}\right)^{2},
\end{aligned}
$$

where $V_{0}=\left(\lambda_{q}+4 \varepsilon_{q}^{2}\right) z_{1}^{2}+z_{2}^{2}-4 \varepsilon_{q} z_{1} z_{2}=z^{T} \mathbf{P} z, \mathbf{P}=$ $\left[\begin{array}{cc}\lambda_{q}+4 \varepsilon_{q}^{2} & -2 \varepsilon_{q} \\ -2 \varepsilon_{q} & 1\end{array}\right]$, and $\alpha_{q r}^{*}, \beta_{q r}^{*}$ are positive constant. It is noted that matrix $\mathbf{P}$ is always positive if $\lambda_{q}>0$ and $\varepsilon_{q}$ is arbitrary real number.

To calculate time derivative of Lyapunov function (38)

$$
\begin{aligned}
\dot{V}\left(z_{1}, z_{2}, \alpha_{q r}, \beta_{q r}\right)= & \mathbf{z}^{T}\left[\bar{A}^{T}\left(z_{1}\right) \mathbf{P}+\mathbf{P} \bar{A}\left(z_{1}\right)\right] \mathbf{z} \\
& +\frac{1}{\delta_{1}}\left(\alpha_{q r}-\alpha_{q r}^{*}\right) \dot{\alpha}_{q r} \\
& +\frac{1}{\delta_{2}}\left(\beta_{q r}-\beta_{q r}^{*}\right) \dot{\beta}_{q r} .
\end{aligned}
$$

Considering formulas (35) and (37)

$$
\dot{V}_{0}=\mathbf{z}^{T}\left[\overline{\mathbf{A}}^{T}\left(z_{1}\right) \mathbf{P}+\mathbf{P} \bar{A}\left(z_{1}\right)\right] \mathbf{z} \leq-\frac{1}{2\left|z_{1}\right|} \mathbf{z}^{T} \mathbf{\Theta} \mathbf{z} .
$$

Considering $\left|\dot{\tilde{a}}_{q}\right| \leq a_{q 1}$ and formula (36), Symmetric matrix $\Theta$ is calculated as

$$
\Theta=\left[\begin{array}{ll}
\Theta_{11} & \Theta_{12} \\
\Theta_{21} & 4 \varepsilon_{q}
\end{array}\right],
$$

where $\Theta_{11}=2 \lambda_{q} \alpha_{q r} \widetilde{b}_{q}+4 \varepsilon_{q} \widetilde{b}_{q}\left(2 \varepsilon_{q} \alpha_{q r}-\beta_{q r}\right)+4 \varepsilon_{q} \rho_{q 2}, \Theta_{12}=$ $\Theta_{21}=\left(\beta_{q r} \widetilde{b}_{q}-2 \varepsilon_{q} \alpha_{q r} \widetilde{b}_{q}-\lambda_{q}-4 \varepsilon_{q}^{2}\right)-\rho_{q 2}$.

To guarantee positive definiteness of matrix $\Theta, \beta_{q r}$ is designed as

$$
\beta_{q r}=2 \varepsilon_{q} \alpha_{q r}
$$

Then, if $\alpha_{q r}$ satisfies

$$
\alpha_{q r}>\frac{\left(2 \chi_{3}+\lambda_{q}+4 \varepsilon_{q}^{2}\right)^{2}}{12 \varepsilon_{q} \lambda_{q}\left(1-\chi_{q 1}\right)}-\frac{\varepsilon_{q}\left(4 \chi_{3}+1\right)}{\lambda_{q}\left(1-\chi_{q 1}\right)}
$$

matrix $\Theta$ is positive definite, and minimum eigenvalue $\lambda_{\text {min }}(\Theta) \geq 2 \varepsilon_{q}$ is satisfied.

In view of formulas (40), (42), and (43) being established, then

$$
\begin{aligned}
\dot{V}_{0} & \leq-\frac{1}{2\left|z_{1}\right|} \mathbf{z}^{T} \Theta \mathbf{z} \leq-\frac{2 \varepsilon_{q}}{2\left|z_{1}\right|} \mathbf{z}^{T} \mathbf{z} \\
& =-\frac{\varepsilon_{q}}{\left|z_{1}\right|}\|\mathbf{z}\|^{2},
\end{aligned}
$$$$
\lambda_{\text {min }}(\mathbf{P})\|\mathbf{z}\|^{2} \leq \mathbf{z}^{T} \mathbf{P} \mathbf{z} \leq \lambda_{\max }(\mathbf{P})\|\mathbf{z}\|^{2},
$$

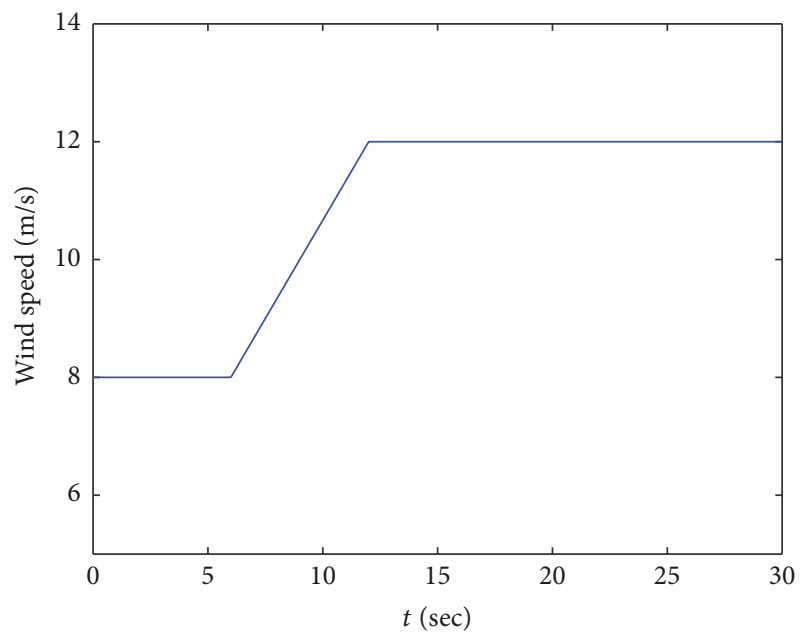

Figure 3: Gradient wind speed.

where $\|\mathbf{z}\|^{2}=z_{1}^{2}+z_{2}^{2}=\left|s_{1}\right|+z_{2}^{2}$, and

$$
\left|z_{1}\right|=\left|s_{1}\right|^{1 / 2} \leq\|\mathbf{z}\| \leq \frac{V_{0}^{1 / 2}(\mathbf{z})}{\lambda_{\min }^{1 / 2}(\mathbf{P})} .
$$

Then

$$
\dot{V}_{0} \leq-r_{q} V_{0}^{1 / 2},
$$

where $r_{q}=\varepsilon_{q} \lambda_{\min }^{1 / 2}(P) / \lambda_{\max }(P)$.

To combine formulas (39) and (46), then

$$
\begin{aligned}
\dot{V}(\mathbf{z}, \alpha, \beta)= & \dot{\mathbf{z}}^{T} \mathbf{P} \mathbf{z}+\mathbf{z}^{T} \mathbf{P} \dot{\mathbf{z}}+\frac{1}{\delta_{1}} \varepsilon_{\alpha} \dot{\alpha}_{q r}+\frac{1}{\delta_{2}} \varepsilon_{\beta} \dot{\beta}_{q r} \\
\leq & -\frac{1}{\left|z_{1}\right|} \mathbf{z}^{T} \boldsymbol{\Theta} \mathbf{z}+\frac{1}{\delta_{1}} \varepsilon_{\alpha} \dot{\alpha}_{q r}+\frac{1}{\delta_{2}} \varepsilon_{\beta} \dot{\beta}_{q r} \\
\leq & -r_{q} V_{0}^{1 / 2}+\frac{1}{\delta_{1}} \varepsilon_{\alpha} \dot{\alpha}_{q r}+\frac{1}{\delta_{2}} \varepsilon_{\beta} \dot{\beta}_{q r} \\
= & -r_{q} V_{0}^{1 / 2}-\frac{\omega_{q 1}}{\sqrt{2 \delta_{1}}}\left|\varepsilon_{\alpha}\right|-\frac{\omega_{q 2}}{\sqrt{2 \delta_{2}}}\left|\varepsilon_{\beta}\right| \\
& +\frac{1}{\delta_{1}} \varepsilon_{\alpha} \dot{\alpha}_{q r}+\frac{1}{\delta_{2}} \varepsilon_{\beta} \dot{\beta}_{q r}+\frac{\omega_{q 1}}{\sqrt{2 \delta_{1}}}\left|\varepsilon_{\alpha}\right| \\
& +\frac{\omega_{q 2}}{\sqrt{2 \delta_{2}}}\left|\varepsilon_{\beta}\right|,
\end{aligned}
$$

where $\varepsilon_{\alpha}=\alpha_{q r}-\alpha_{q r}^{*} \varepsilon_{\beta}=\beta_{q r}-\beta_{q r}^{*}$.

Considering inequation $\left(a^{2}+b^{2}+c^{2}\right)^{1 / 2} \leq|a|+|b|+|c|$ and formula (38), then

$$
\begin{aligned}
& -r_{q} V_{0}^{1 / 2}-\frac{\omega_{q 1}}{\sqrt{2 \delta_{1}}}\left|\varepsilon_{\alpha}\right|-\frac{\omega_{q 2}}{\sqrt{2 \delta_{2}}}\left|\varepsilon_{\beta}\right| \\
& \leq-\eta_{q 0} \sqrt{V\left(\mathbf{z}, \alpha_{q r}, \beta_{q r}\right)},
\end{aligned}
$$


where $\eta_{q 0}=\min \left(r_{q}, \omega_{q 1}, \omega_{q 2}\right)$. Then

$$
\begin{aligned}
\dot{V}\left(z_{1}, z_{2}, \alpha_{q r}, \beta_{q r}\right) \leq & -\eta_{q 0} \sqrt{V\left(z_{1}, z_{2}, \alpha_{q r}, \beta_{q r}\right)} \\
& +\frac{1}{\delta_{1}} \varepsilon_{\alpha} \dot{\alpha}_{q r}+\frac{1}{\delta_{2}} \varepsilon_{\beta} \dot{\beta}_{q r} \\
& +\frac{\omega_{q 1}}{\sqrt{2 \delta_{1}}}\left|\varepsilon_{\alpha}\right|+\frac{\omega_{q 2}}{\sqrt{2 \delta_{2}}}\left|\varepsilon_{\beta}\right| .
\end{aligned}
$$

One solution of formula (32) is

$$
\alpha_{q r}=\alpha_{q r}(0)+\omega_{q 1} \sqrt{\frac{\delta_{1}}{2}} \cdot t \quad 0 \leq t \leq t_{f} .
$$

Hence, $\alpha_{q r}, \beta_{q r}$ are both bounded; namely, $\alpha_{q r}-\alpha_{q r}^{*}<0$, $\beta_{q r}-\beta_{q r}^{*}<0$. Then formula (49) is expressed as

$$
\begin{aligned}
\dot{V}\left(z_{1}, z_{2}, \alpha_{q r}, \beta_{q r}\right) \leq & -\eta_{q 0}\left[V\left(z_{1}, z_{2}, \alpha_{q r}, \beta_{q r}\right)\right]^{1 / 2} \\
& -\left|\varepsilon_{\alpha}\right|\left(\frac{1}{\delta_{1}} \dot{\alpha}_{q r}-\frac{\omega_{q 1}}{\sqrt{2 \delta_{1}}}\right) \\
& -\left|\varepsilon_{\beta}\right|\left(\frac{1}{\delta_{2}} \dot{\beta}_{q r}-\frac{\omega_{q 2}}{\sqrt{2 \delta_{2}}}\right), \\
\dot{V}\left(z_{1}, z_{2}, \alpha_{q r}, \beta_{q r}\right) \leq & -\eta_{q 0}\left[V\left(z_{1}, z_{2}, \alpha_{q r}, \beta_{q r}\right)\right]^{1 / 2} \\
& +\xi_{q},
\end{aligned}
$$

where $\xi_{q}=-\left|\varepsilon_{\alpha}\right|\left(\left(1 / \delta_{1}\right) \dot{\alpha}_{q r}-\omega_{q 1} / \sqrt{2 \delta_{1}}\right)-\left|\varepsilon_{\beta}\right|\left(\left(1 / \delta_{2}\right) \dot{\beta}_{q r}-\right.$ $\left.\omega_{q 2} / \sqrt{2 \delta_{2}}\right)$.

$\left(\mathbf{C}_{1}\right)$ Provided that $t \geq 0$, we have $\left|s_{1}\right|>\mu_{q}$ and $\alpha_{q r}>\alpha_{q r m}$. Then considering formula (32)

$$
\begin{aligned}
& \dot{\alpha}_{q r}=\omega_{q 1} \sqrt{\frac{\delta_{1}}{2}}, \\
& \xi_{q}=-\left|\varepsilon_{\beta}\right|\left(\frac{1}{\delta_{2}} \dot{\beta}_{q r}-\frac{\omega_{q 2}}{\sqrt{2 \delta_{2}}}\right) .
\end{aligned}
$$

To choose $\varepsilon_{q}=\left(\omega_{q 2} / \omega_{q 1}\right) \sqrt{\delta_{2} / \delta_{1}}$ and combine formula (42), then

$$
\dot{\beta}_{q r}=\omega_{q 2} \sqrt{\frac{\delta_{2}}{2}}
$$

then $\xi_{q}=0$, and

$$
\dot{V}\left(z_{1}, z_{2}, \alpha_{q r}, \beta_{q r}\right) \leq-\eta_{q 0}\left[V\left(z_{1}, z_{2}, \alpha_{q r}, \beta_{q r}\right)\right]^{1 / 2} .
$$

Take note that $\alpha_{q r}$ increases according to $\dot{\alpha}_{q r}=\omega_{q 1} \sqrt{\delta_{1} / 2}$. When formula (22) is satisfied, positive definiteness of matrix $\Theta$ and formula (55) are guaranteed. Then after finite time $t_{f}$, $\left|s_{1}\right| \leq \mu_{q}$ is established.
$\left(\mathbf{C}_{2}\right)$ To suppose $\left|s_{1}\right|<\mu_{q}$, then according to formula (32), the formula

$$
\dot{\alpha}_{q r}= \begin{cases}-\omega_{q 1} \sqrt{\frac{\delta_{1}}{2}} & \text { if } \alpha_{q r}>\alpha_{q r m} \\ \eta_{q} & \text { if } \alpha_{q r} \leq \alpha_{q r m}\end{cases}
$$

and formula (57) may become positive number:

$$
\begin{aligned}
& \xi_{q} \\
& = \begin{cases}2\left|\alpha_{q r}-\alpha_{q r}^{*}\right| \frac{\omega_{q 1}}{\sqrt{2 \delta_{1}}} & \text { if } \alpha_{q r}>\alpha_{q r m} \\
-\left|\alpha_{q r m}-\alpha_{a r}^{*}+\eta_{q} \cdot t\right|\left(\frac{\eta_{q}}{\delta_{1}}-\frac{\omega_{q 1}}{\sqrt{2 \delta_{1}}}\right) & \text { if } \alpha_{q r} \leq \alpha_{q r m} .\end{cases}
\end{aligned}
$$

It is worth noting that the expression under $\alpha_{q r} \leq \alpha_{q r m}$ is effective in finite time, because as long as $\alpha_{q r}<\alpha_{q r m}$ is valid, $\alpha_{q r}$ will increase according to $\alpha_{q r}=\alpha_{q r m}+\eta_{q} \cdot t$, and then the other expression of formula (57) is effective.

The symbol $\dot{V}\left(z_{1}, z_{2}, \alpha_{q r}, \beta_{q r}\right)$ in formula (52) becomes uncertain in view of formula (57). $\left|s_{1}\right|$ may be greater than $\mu_{q}$ due to the diminution of control gain $\alpha_{q r}$ and $\beta_{q r}$. Once $\left|s_{1}\right|$ is greater than $\mu_{q}, C_{1}$ is established. Hence, $s_{1}$ reaches $\left|s_{1}\right| \leq \mu_{q}$ in finite time during adaptive process and then leaves this range and always stays in a bigger one $\left|s_{1}\right| \leq \eta_{q 1}, \eta_{q 1}>\mu_{q}$.

When $\left|s_{1}\right| \leq \mu_{q}$ is satisfied, $\left|\dot{s}_{1}\right|$ can be estimated via formulas (29) and (32):

$$
\begin{aligned}
\left|\dot{s}_{1}\right| \leq & \left(\left(1-\delta_{1}\right) \alpha_{q r}\right) \mu_{q}^{1 / 2} \\
& +\left[\varepsilon_{q}\left(1-\delta_{1}\right) \alpha_{q r}+\chi_{q 3}\right]\left(t_{2}-t_{1}\right)=\bar{\eta}_{q 2},
\end{aligned}
$$

where $t_{1}$ and $t_{2}$ are the moment entering into and leaving $\left|s_{1}\right| \leq \mu_{q}$. When $\mu_{q} \leq\left|s_{1}\right| \leq \eta_{q 1}$

$$
\begin{aligned}
& \left|\dot{s}_{1}\right| \leq\left(1-\delta_{1}\right)\left(\eta_{q 1}^{1 / 2}+\varepsilon_{q}\right)\left(\alpha_{q r}\left(t_{2}\right)+\omega_{q 1} \sqrt{\frac{\eta_{q 1} \delta_{1}}{2}}\right) \\
& \cdot\left(t_{3}-t_{2}\right)+\chi_{3}\left(t_{3}-t_{2}\right)=\tilde{\eta}_{q 2},
\end{aligned}
$$

where $t_{2}$ and $t_{3}$ are the moment entering into and leaving $\left|s_{1}\right| \leq \mu_{q}$. From formulas (58) and (59)

$$
\left|\dot{s}_{1}\right| \leq \max \left(\bar{\eta}_{q 2}, \widetilde{\eta}_{q 2}\right)=\eta_{q 2}
$$

Namely, $s_{1}$ can enter into $W=\left\{s_{1}, \dot{s}_{1}:\left|s_{1}\right| \leq \eta_{q 1},\left|\dot{s}_{1}\right| \leq\right.$ $\left.\eta_{q 2}, \eta_{q 1}>\mu_{q}\right\}$ in finite time and stay here. Theorem 1 is proved.

\section{Case Studies}

Simulation verification of the proposed control scheme is carried out based on MATLAB platform. Nominal parameters of doubly fed wind turbine system are $\omega_{s}=2 \pi 60 \mathrm{rad} / \mathrm{s}, V_{s}=$ $460 \sqrt{2 / 3} \mathrm{~V}, L_{s}=35.5 \mathrm{mH}, L_{r}=35.5 \mathrm{mH}, L_{m}=35.7 \mathrm{mH}$, $R_{r}=228 \mathrm{~m} \Omega, R_{s}=82 \mathrm{~m} \Omega, p=2, J=3.662 \mathrm{Kgm}^{2}, K_{g b}=25$, $R=7.3 \mathrm{~m}, C_{p \max }=0.4$, and $\lambda_{\text {opt }}=7.5$. The choice of control 


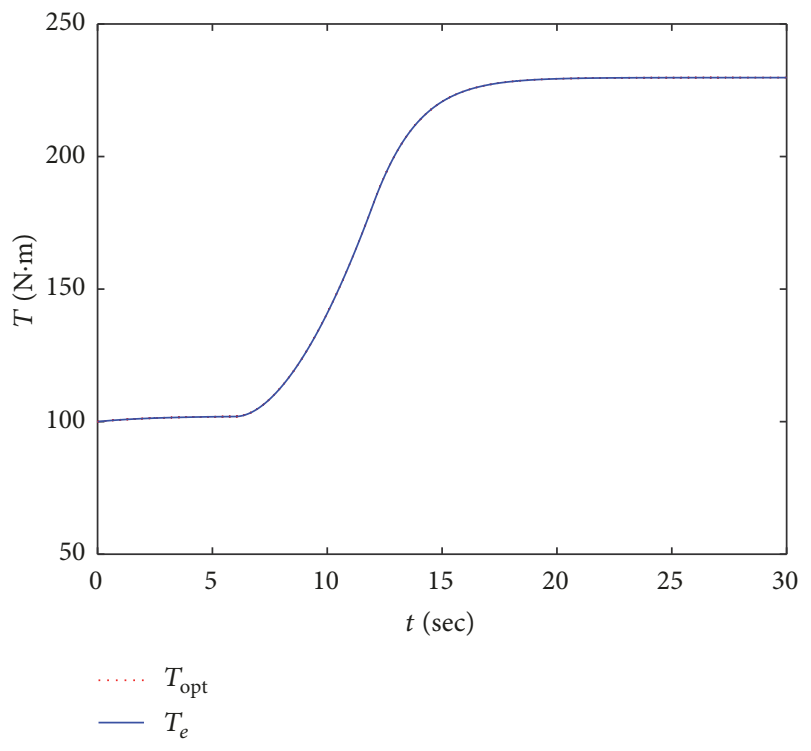

Figure 4: Optimal torque tracking under fixed gain.

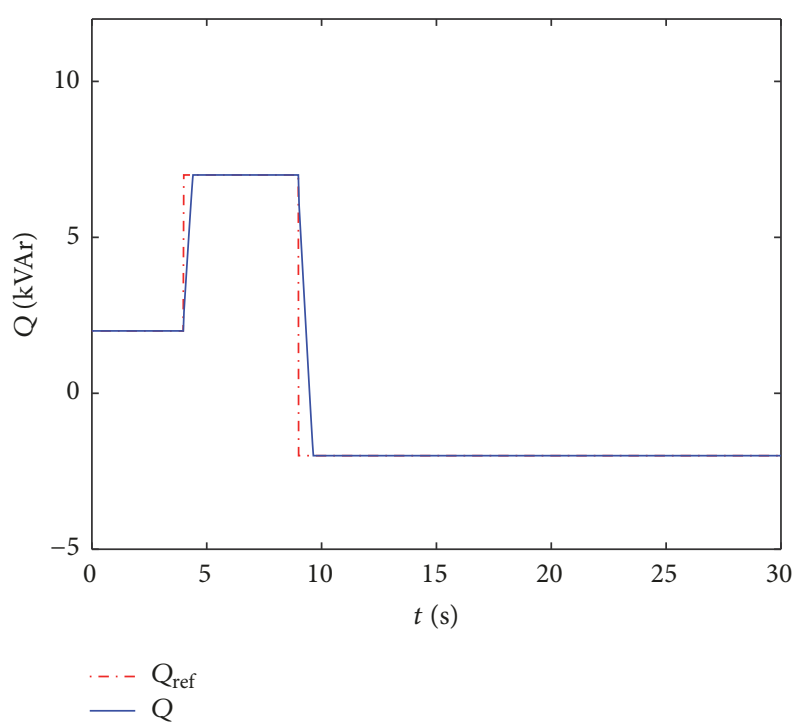

FIGURE 5: Reactive power regulation under fixed gain.

parameters is according to formulas (19) to (21) and formula (32) and combines actual simulation experiment. The related fixed gain control parameters are $\alpha_{1}=4.5, \beta_{1}=40, \alpha_{2}=0.1$, $\beta_{2}=5, U_{m 1}=300, U_{m 2}=300$, and the adaptive gain parameters are $\omega_{q 1}=2, \delta_{1}=2, \mu_{q}=0.2, \eta_{q 1}=1, \varepsilon_{q 1}=5$, $\alpha_{\text {qrm }}=0.5, \omega_{d 1}=2, \delta_{d 2}=2, \mu_{d}=0.2, \eta_{d}=1, \varepsilon_{d 1}=10$, $\alpha_{d r m}=0.2$.

Case 1 (gradient wind). Gradient wind speed model can be described by formulas (61). To choose $t_{1 G}=6 \mathrm{~s}, t_{2 G}=12 \mathrm{~s}$ and $\max R=4$

$$
v_{w}= \begin{cases}v_{b} & t<t_{1 G} \\ v_{\text {ramp }} & t_{1 G}<t<t_{1 G}+t_{2 G} \\ \max R & t>t_{2 G}\end{cases}
$$

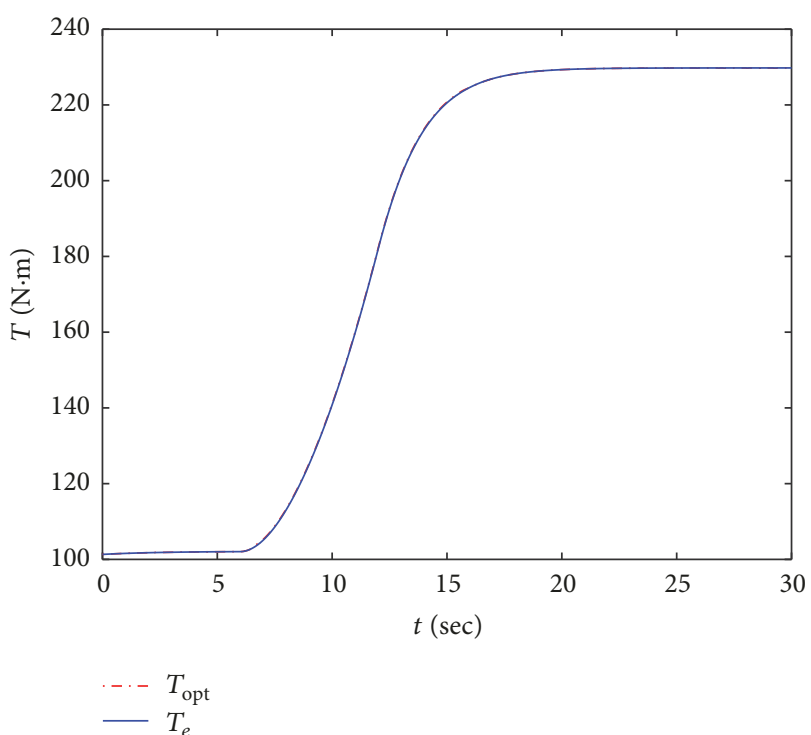

FIGURE 6: Optimal torque tracking under adaptive gain.

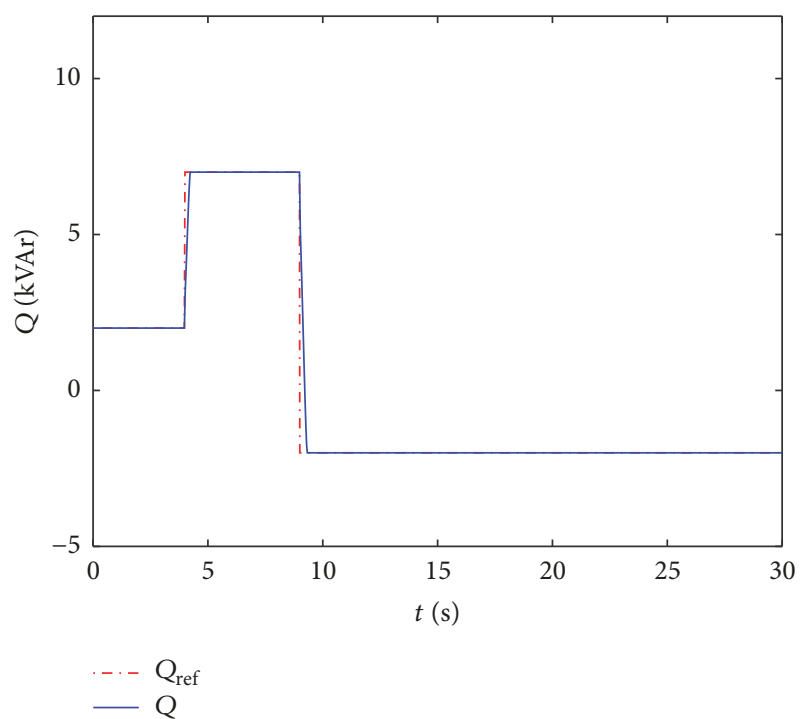

FIGURE 7: Reactive power regulation under adaptive gain.

$$
v_{\text {ramp }}=\max R\left(1-\frac{t-t_{2 R}}{t_{1 R}-t_{2 R}}\right) .
$$

Figure 3 shows gradient wind speed variation. The curves of optimal tracking torque and reactive power regulation under fixed control gain scheme are shown in Figures 4 and 5. It is observed that the two control objectives can be achieved, but the uncertainty upper bound is not easy to measure in actual wind power system. Therefore, adaptive gain secondorder sliding mode control scheme is put into effect. Maximum wind power tracking and reactive power regulation effect can also be achieved under adaptive control gain scheme as shown in Figures 6 and 7. Figure 8 shows rotor control voltages which indicate chattering reduction. Figure 9 


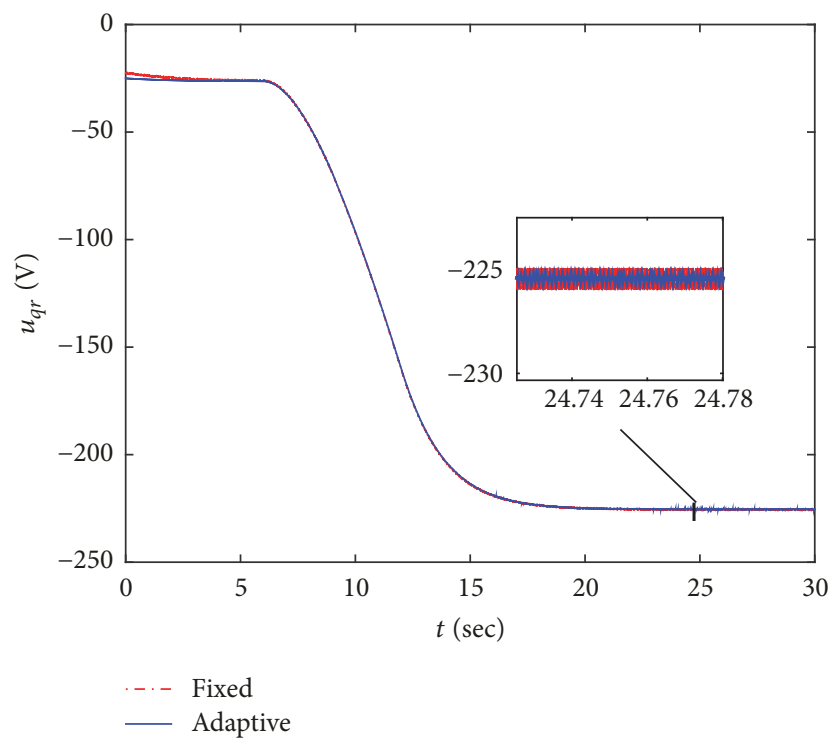

FIGURE 8: Rotor voltage in $q$-axis.

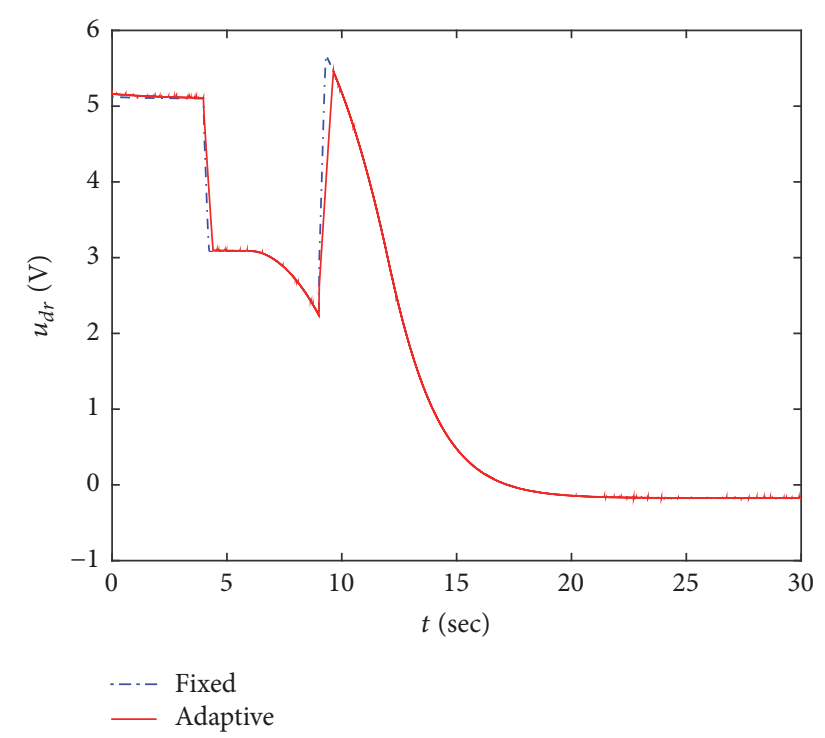

FIgURE 9: Rotor voltage in $d$-axis.

is $d$-axis rotor voltage and Figure 10 shows $d-q$ axis rotor current. Control gain regulation according to wind speed variation is shown in Figure 11.

Case 2 (random wind). The random wind speed variation is shown in Figure 12. Considering system uncertainties, coefficients of mutual induction $L_{m}$ and rotor resistance fluctuate $\pm 10 \%$. Grid voltage and grid frequency fluctuation are \pm 10 and $\pm 20 \%$, respectively. For highlighting the controller performances, the proposed AGSOSM (adaptive gain secondorder sliding mode) method and ASOSM (adaptive secondorder sliding mode) controller [25] are, respectively, executed on the wind turbine system. The related ASOSM controller parameters are referred to in [25]. Figure 13 shows that the actual electromagnetic torque can rapidly track desired value, and Figure 14 shows that reactive power regulation can still

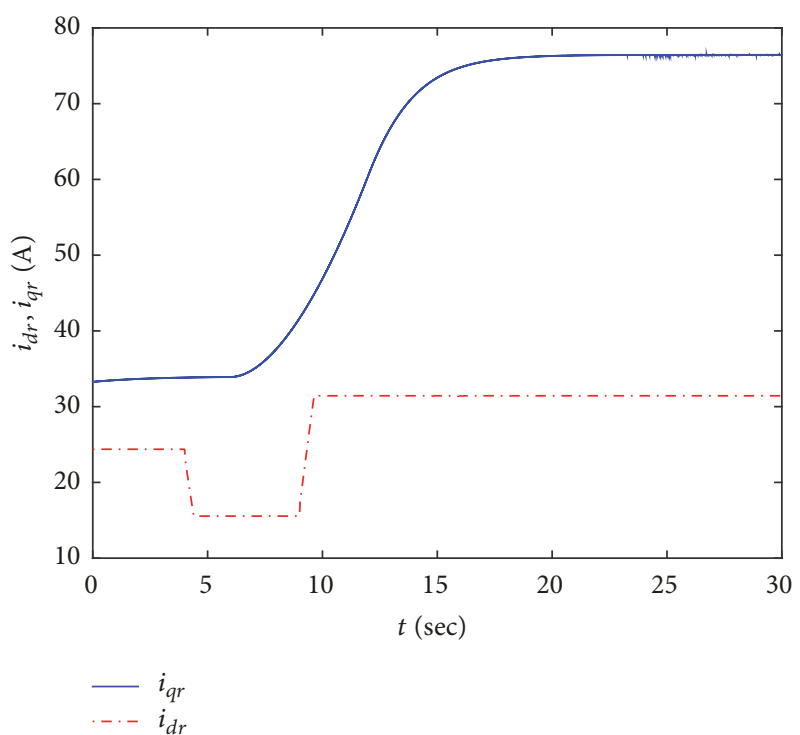

FIGURE 10: Rotor current in $d q$-axis under adaptive gain.

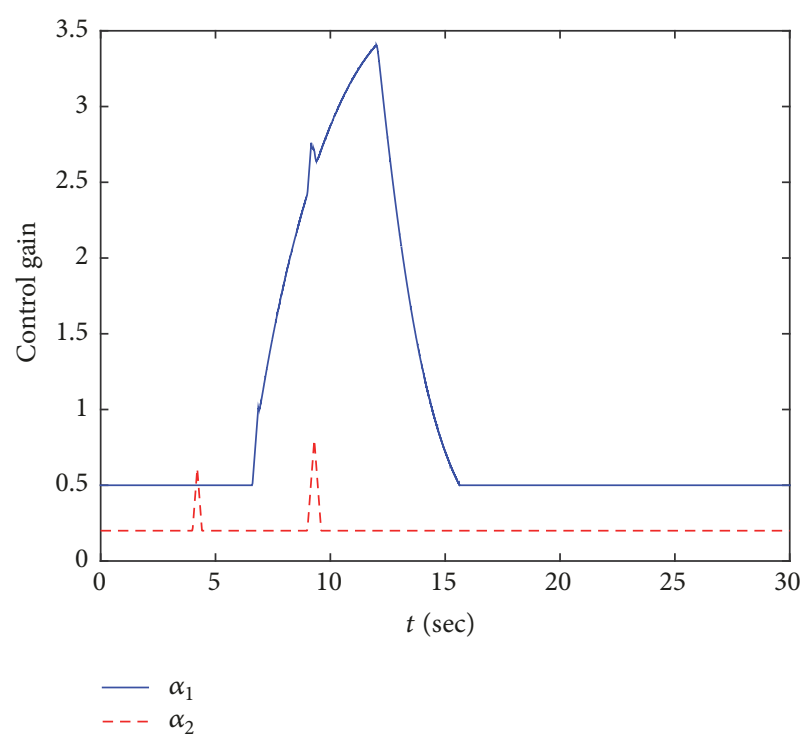

FIGURE 11: Control gain under adaptive gain.

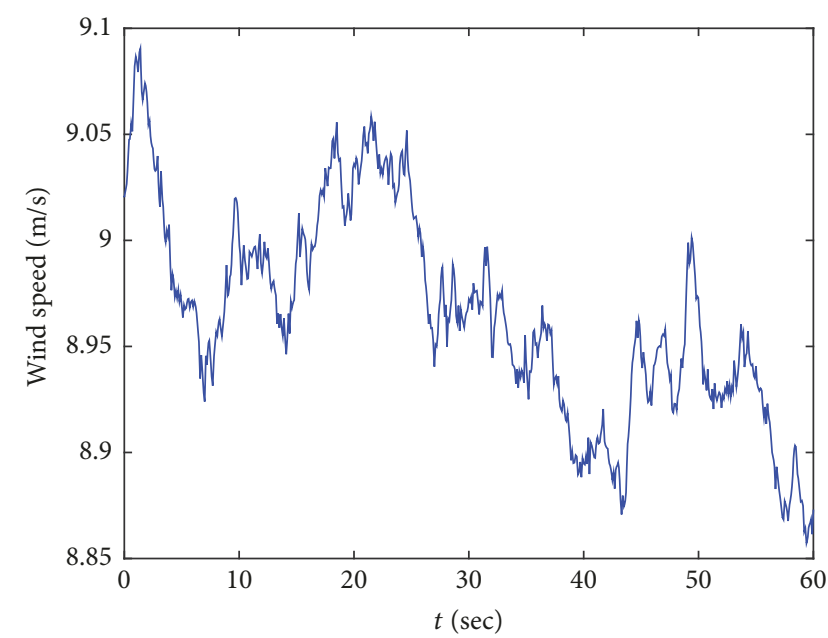

FIGURE 12: Random wind speed. 


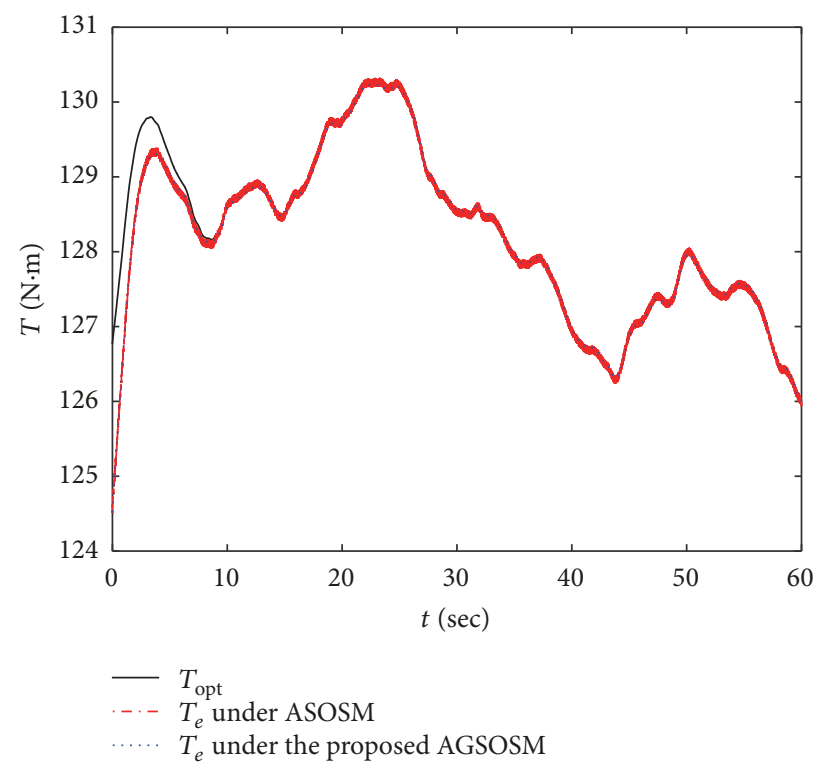

FIGURE 13: Optimal torque tracking.

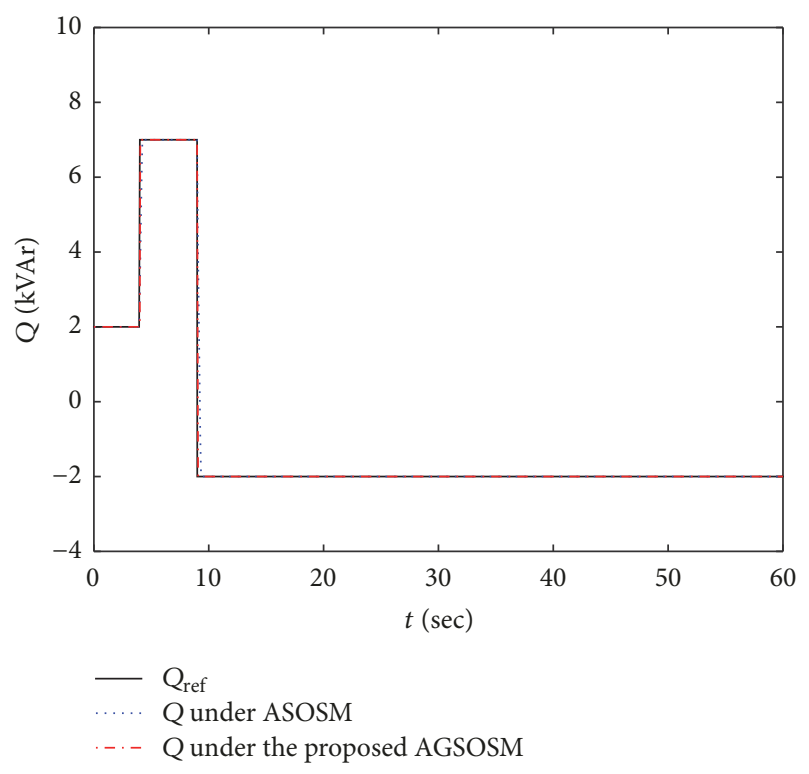

FIGURE 14: Reactive power regulation.

satisfy the demand under random wind under the two control schemes. However, the proposed scheme can achieve higher control accuracy. As is shown in Figure 15, the rotor control voltage chattering is greatly alleviated under the proposed control method. Rotor current under $d q$-axis is shown in Figure 16.

\section{Conclusions}

For achieving maximum wind power tracking and reactive power regulation of doubly fed wind turbine system, this paper proposed an adaptive gain second-order sliding mode control scheme. Firstly, the system model is established.
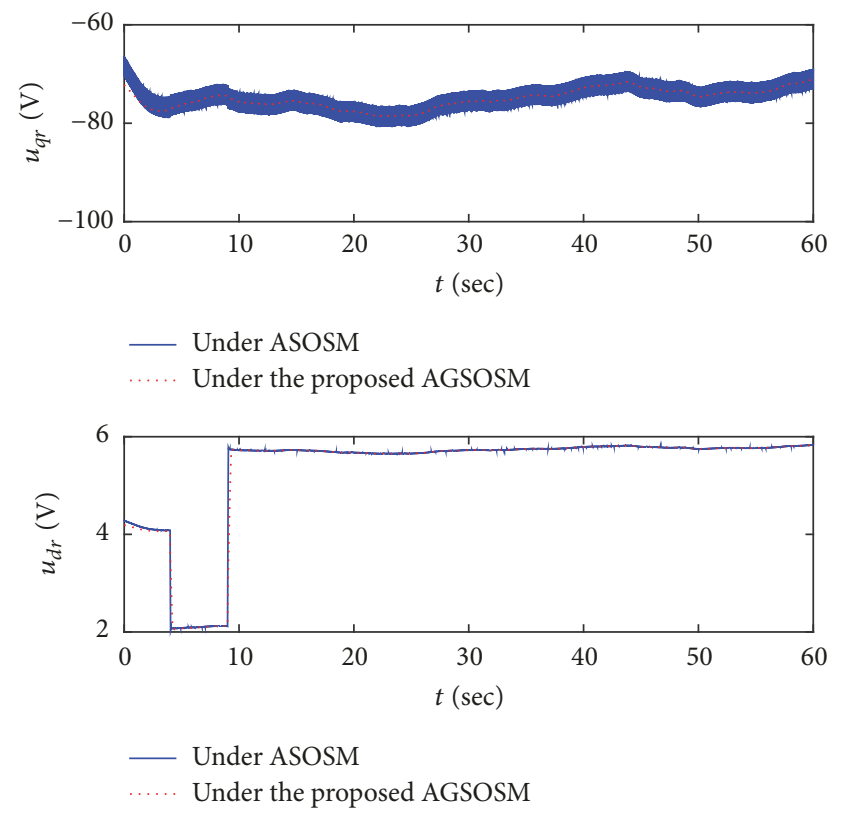

FIGURE 15: Rotor voltage in $d q$-axis.
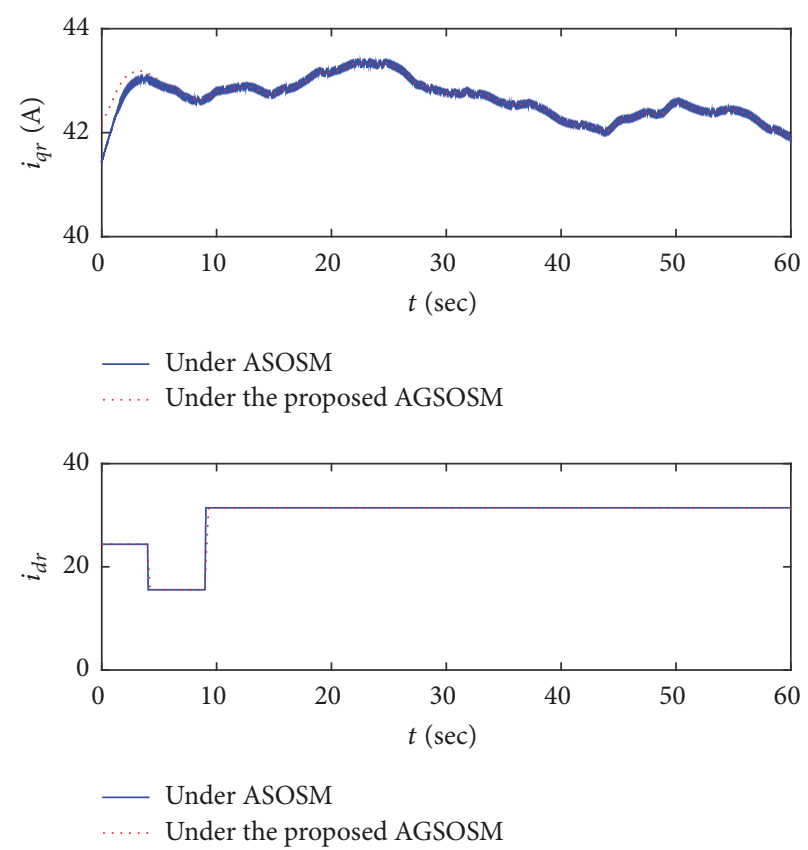

FIGURE 16: Rotor current in $d q$-axis.

Then second-order sliding mode rotor voltage controllers are designed after detailed description of the two control objectives. Considering the circumstance of unknown uncertainty upper bound, adaptive gain second-order sliding mode rotor voltages controllers are designed, and finite time stability is proved in detail. Finally, simulation experiments under gradient wind and random wind verify that the proposed control strategy can achieve optimal torque tracking and reactive power regulation and is robust to parameter uncertainties such as network voltage, network frequency, rotor 
resistance, and mutual inductance. The proposed control method also makes rotor control voltage continuous and the control chattering is greatly restrained.

\section{Conflicts of Interest}

The authors declare that they have no conflicts of interest.

\section{Acknowledgments}

This work was supported by National Natural Science Foundation of China under Grants 61375084 and 61773015, Doctoral Scientific Research Initial Foundation of Shandong Jiaotong University, Jinan Science and Technology Innovation Program of Shandong, China, under Grant 201401203, and Key Program of Natural Science Foundation of Shandong Province ZR2015QZ08.

\section{References}

[1] A. Dimov and S. Bolik, "Wind turbine manufacturers observation regarding reactive power support and control requirements," IET Renewable Power Generation, vol. 11, no. 4, pp. 539544, 2017.

[2] C. L. Archer, H. P. Simão, W. Kempton, W. B. Powell, and M. J. Dvorak, "The challenge of integrating offshore wind power in the U.S. electric grid. Part I: Wind forecast error," Journal of Renewable Energy, vol. 103, pp. 346-360, 2017.

[3] Z. Chen, M. Yin, Y. Zou, K. Meng, and Z. Dong, "Maximum Wind Energy Extraction for Variable Speed Wind Turbines with Slow Dynamic Behavior," IEEE Transactions on Power Systems, vol. 32, no. 4, pp. 3321-3322, 2017.

[4] S. Taraft, D. Rekioua, D. Aouzellag, and S. Bacha, "A proposed strategy for power optimization of a wind energy conversion system connected to the grid," Energy Conversion and Management, vol. 101, pp. 489-502, 2015.

[5] A. M. Kassem, K. M. Hasaneen, and A. M. Yousef, "Dynamic modeling and robust power control of DFIG driven by wind turbine at infinite grid," International Journal of Electrical Power \& Energy Systems, vol. 44, no. 1, pp. 375-382, 2013.

[6] K. Belmokhtar, M. L. Doumbia, and K. Agbossou, "Novel fuzzy logic based sensorless maximum power point tracking strategy for wind turbine systems driven DFIG (doubly-fed induction generator)," Energy, vol. 76, pp. 679-693, 2014.

[7] H. Jafarnejadsani, J. Pieper, and J. Ehlers, "Adaptive control of a variable-speed variable-pitch wind turbine using radial-basis function neural network," IEEE Transactions on Control Systems Technology, vol. 21, no. 6, pp. 2264-2272, 2013.

[8] F. Fateh, W. N. White, and D. Gruenbacher, "A Maximum Power Tracking Technique for Grid-Connected DFIG-Based Wind Turbines," IEEE Journal of Emerging and Selected Topics in Power Electronics, vol. 3, no. 4, pp. 957-966, 2015.

[9] R. K. Patnaik and P. K. Dash, "Fast adaptive back-stepping terminal sliding mode power control for both the rotor-side as well as grid-side converter of the doubly fed induction generator-based wind farms," IET Renewable Power Generation, vol. 10, no. 5, pp. 598-610, 2016.

[10] N. H. Saad, A. A. Sattar, and A. E.-A. M. Mansour, "Low voltage ride through of doubly-fed induction generator connected to the grid using sliding mode control strategy," Journal of Renewable Energy, vol. 80, pp. 583-594, 2015.
[11] Y.-T. Weng and Y.-Y. Hsu, "Sliding mode regulator for maximum power tracking and copper loss minimisation of a doubly fed induction generator," IET Renewable Power Generation, vol. 9, no. 4, pp. 297-305, 2014.

[12] S. Abdeddaim and A. Betka, "Optimal tracking and robust power control of the DFIG wind turbine," International Journal of Electrical Power \& Energy Systems, vol. 49, no. 1, pp. 234-242, 2013.

[13] Y. Han and X. Liu, "Continuous higher-order sliding mode control with time-varying gain for a class of uncertain nonlinear systems," ISA Transactions ${ }^{\circledR}$, vol. 62, pp. 193-201, 2016.

[14] F. alenciaga and C. A. Evangelista, "2-Sliding active and reactive power control of a wind energy conversion system," IET Control Theory and Applications, vol. 4, no. 11, pp. 2479-2490, 2010.

[15] C. A. Evangelista, F. Valenciaga, and P. Puleston, "Multivariable 2-sliding mode control for a wind energy system based on a double fed induction generator," International Journal of Hydrogen Energy, vol. 37, no. 13, pp. 10070-10075, 2012.

[16] C. Evangelista, F. Valenciaga, and P. Puleston, "Active and reactive power control for wind turbine based on a MIMO 2Sliding mode algorithm with variable gains," IEEE Transactions on Energy Conversion, vol. 28, no. 3, pp. 682-689, 2013.

[17] B. Beltran, M. E. H. Benbouzid, and T. Ahmed-Ali, "Secondorder sliding mode control of a doubly fed induction generator driven wind turbine," IEEE Transactions on Energy Conversion, vol. 27, no. 2, pp. 261-269, 2012.

[18] X. Liu, Y. Han, and C. Wang, "Second-order sliding mode control for power optimisation of DFIG-based variable speed wind turbine," IET Renewable Power Generation, vol. 11, no. 2, pp. 408-418, 2016.

[19] M. I. Martinez, A. Susperregui, and G. Tapia, "Second-order sliding-mode-based global control scheme for wind turbinedriven DFIGs subject to unbalanced and distorted grid voltage," IET Electric Power Applications, vol. 11, no. 6, pp. 1013-1022, 2017.

[20] C. Evangelista, P. Puleston, F. Valenciaga, and L. M. Fridman, "Lyapunov-designed super-twisting sliding mode control for wind energy conversion optimization," IEEE Transactions on Industrial Electronics, vol. 60, no. 2, pp. 538-545, 2012.

[21] P. K. Dash and R. K. Patnaik, "Adaptive second order sliding mode control of doubly fed induction generator in wind energy conversion system," Journal of Renewable and Sustainable Energy, vol. 6, no. 5, Article ID 4899193, 2014.

[22] C. A. Evangelista, A. Pisano, P. Puleston, and E. Usai, "Receding Horizon Adaptive Second-Order Sliding Mode Control for Doubly-Fed Induction Generator Based Wind Turbine," IEEE Transactions on Control Systems Technology, vol. 25, no. 1, pp. 73-84, 2017.

[23] M. Pucci and M. Cirrincione, "Neural MPPT control of wind generators with induction machines without speed sensors," IEEE Transactions on Industrial Electronics, vol. 58, no. 1, pp. 3747, 2011.

[24] J. Hu and X. Yuan, "VSC-based direct torque and reactive power control of doubly fed induction generator," Journal of Renewable Energy, vol. 40, no. 1, pp. 13-23, 2014.

[25] Y. Shtessel, C. Edwards, L. Fridman et al., Sliding Mode Control and Observation, Birkhäuser, New York, NY, USA, 2014.

[26] A. Chalanga, S. Kamal, L. M. Fridman, B. Bandyopadhyay, and J. A. Moreno, "Implementation of Super-Twisting Control: Super-Twisting and Higher Order Sliding-Mode ObserverBased Approaches," IEEE Transactions on Industrial Electronics, vol. 63, no. 6, pp. 3677-3685, 2016. 


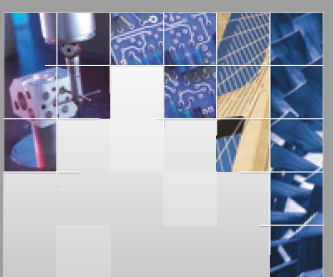

\section{Enfincering}
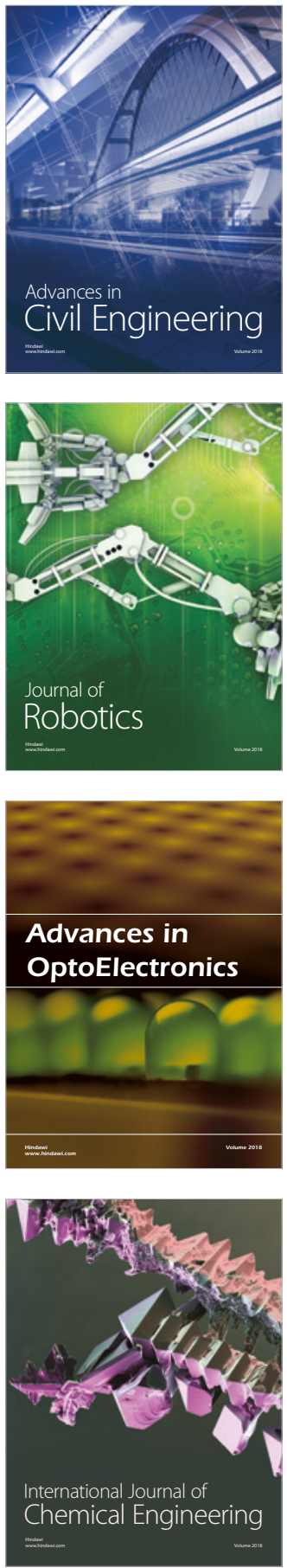

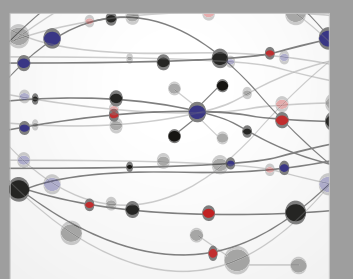

\section{Rotating \\ Machinery}

The Scientific World Journal

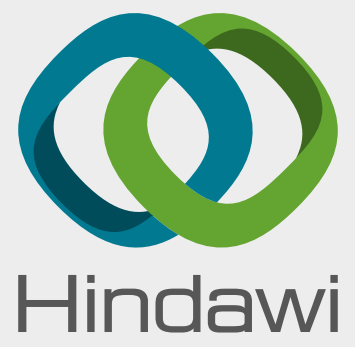

Submit your manuscripts at

www.hindawi.com
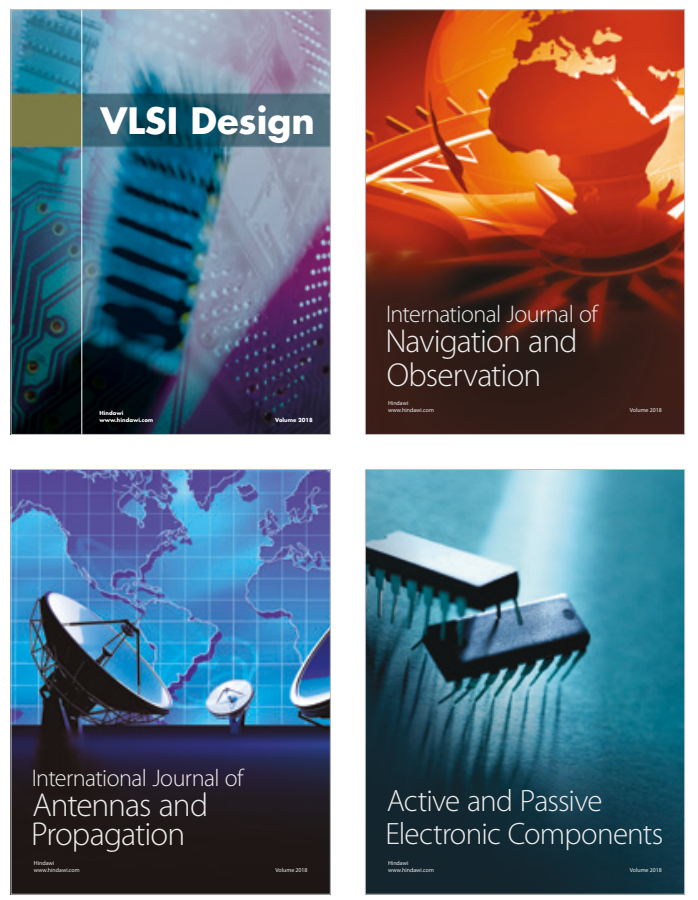
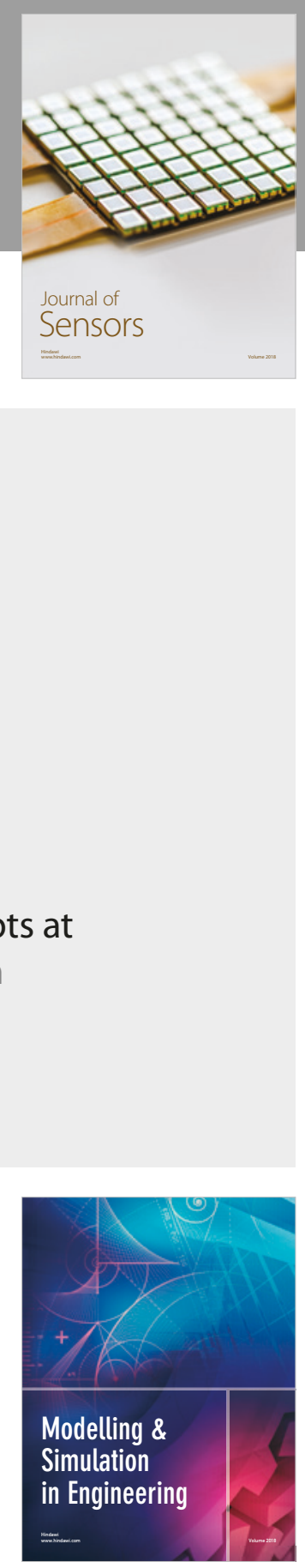

\section{Advances \\ Multimedia}
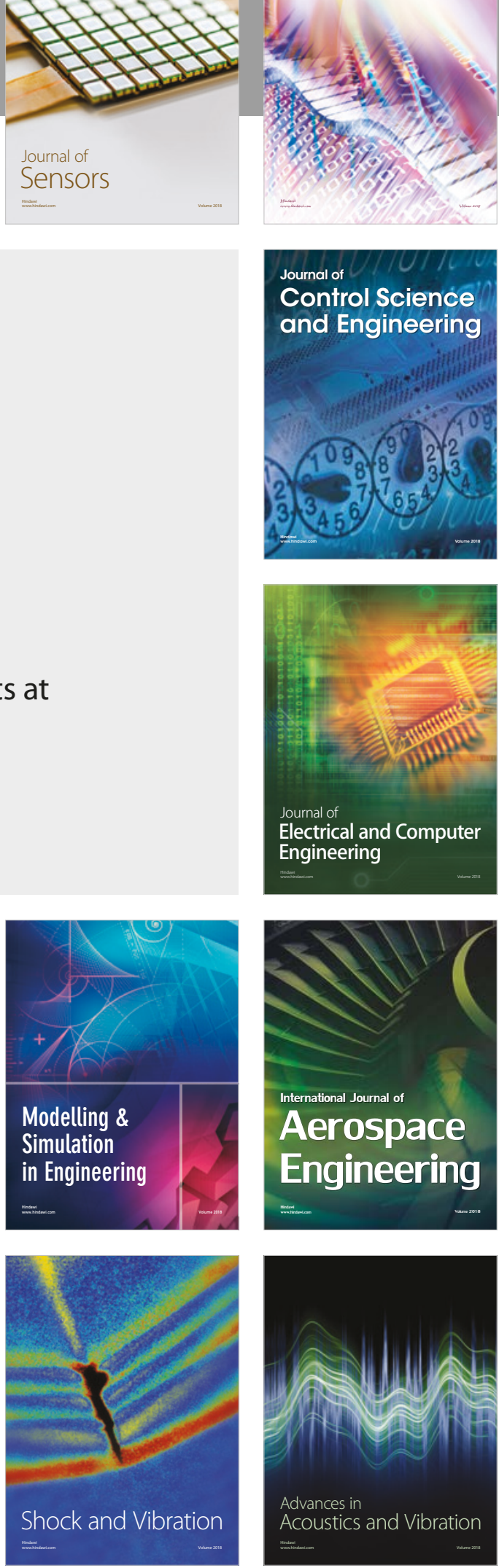